\title{
Evaluation of outcomes following subtotal colectomy for the treatment of idiopathic megacolon in cats
}

Rachel M. Grossman DVM

Julia P. Sumner BVSC

Daniel J. Lopez DVM

Josephine A. Dornbusch DVM

Ameet Singh DVM, DVSC

Cassie N. Lux DVM

Susannah J. Sample DVM, PhD

Julius M. Liptak BVSc, MVetClinStud

Janet A. Grimes DVM, MS

David A. Upchurch DVM, MS

Michael S. Blumenthal DVM

Marine Traverson DVM, MS

Nicole J. Buote DVM

Sarah J. Marvel DVM, MS

Michelle A. Steffey DVM

Shiori Arai DVM, PhD

Jeffrey P. Little DVM

Aylin Atilla VMD, MS

Jennifer L. Huck DVM

Kathryn A. Pitt DVM, MS

From the Department of Clinical Sciences, College of Veterinary Medicine, Cornell University, Ithaca, NY 14853 (Grossman, Sumner, Lopez); Department of Clinical Sciences, College of Veterinary Medicine, The Ohio State University, Columbus, $\mathrm{OH} 43210$ (Dornbusch); Department of Clinical Studies, Ontario Veterinary College, University of Guelph, Guelph, ON NIG 2WI, Canada (Singh); Department of Small Animal Clinical Sciences, College of Veterinary Medicine, University of Tennessee, Knoxville, TN 37996 (Lux); Department of Surgical Sciences, School of Veterinary Medicine, University of Wisconsin, Madison, WI 53706 (Sample); VCA Alta Vista Animal Hospital, Ottawa, ON KIT IM9, Canada (Liptak); Department of Small Animal Medicine and Surgery, College of Veterinary Medicine, University of Georgia, Athens, GA 30602 (Grimes); Department of Clinical Sciences, College of Veterinary Medicine, Kansas State University, Manhattan, KS 66506 (Upchurch); Department of Small Animal Clinical Sciences, College of Veterinary Medicine, University of Florida, Gainesville, FL 32608 (Blumenthal); Department of Clinical Sciences, College of Veterinary Medicine, North Carolina State University, Raleigh, NC 27607 (Traverson); VCA West Los Angeles Animal Hospital, Los Angeles, CA 90025 (Buote); Department of Clinical Sciences, College of Veterinary Medicine and Biomedical Sciences, Colorado State University, Fort Collins, CO 80523 (Marvel); Department of Surgical and Radiological Sciences, School of Veterinary Medicine, University of California-Davis, Davis, CA 95616 (Steffey); Department of Clinical Sciences, College of Veterinary Medicine, University of Minnesota, Saint Paul, MN 55108 (Arai); Veterinary Referral Surgical Practice, Roswell, GA 30076 (Little); Department of Veterinary Clinical and Diagnostic Services, Faculty of Veterinary Medicine, University of Calgary, AB T3C 0 8, Canada (Atilla); VCA Canada Western Veterinary Specialist and Emergency Centre, Calgary, $A B$ T3C 0 8, Canada (Atilla); Department of Clinical Sciences and Advanced Medicine, School of Veterinary Medicine, University of Pennsylvania, Philadelphia, PA 19104 (Huck); Department of Small Animal Clinical Sciences, College of Veterinary Medicine, Michigan State University, East Lansing, Ml 48824 (Pitt).

Address correspondence to Dr. Sumner (jsumner@cornell. edu).

\section{OBJECTIVE}

To evaluate outcomes in cats undergoing subtotal colectomy for the treatment of idiopathic megacolon and to determine whether removal versus nonremoval of the ileocecocolic junction (ICJ) was associated with differences in outcome.

\section{ANIMALS}

166 client-owned cats.

\section{PROCEDURES}

For this retrospective cohort study, medical records databases of 18 participating veterinary hospitals were searched to identify records of cats with idiopathic megacolon treated by subtotal colectomy from January 2000 to December 2018. Data collection included perioperative and surgical variables, complications, outcome, and owner perception of the procedure. Data were analyzed for associations with outcomes of interest, and Kaplan-Meier survival time analysis was performed.

\section{RESULTS}

Major perioperative complications occurred in $9.9 \%$ (I5/I5I) of cats, and $14 \%$ (12/87) of cats died as a direct result of treatment or complications of megacolon. The median survival time was not reached. Cats with (vs without) a body condition score < 4/9 (hazard ratio [HR], 5.97), preexisting heart disease (HR, 3.2I), major perioperative complications (HR, 27.8), or long-term postoperative liquid feces (HR, 10.4) had greater hazard of shorter survival time. Constipation recurrence occurred in 32\% (24/74) of cats at a median time of 344 days and was not associated with retention versus removal of the ICJ; however, ICJ removal was associated with long-term liquid feces (OR, 3.45), and a fair or poor outcome on owner assessment (OR, 3.6).

\section{CONCLUSIONS AND CLINICAL RELEVANCE}

Results indicated that subtotal colectomy was associated with long survival times and a high rate of owner satisfaction. Removal of the ICJ was associated with less favorable outcomes in cats of the present study.

M egacolon is a term used to describe the end point of severe, intractable constipation leading to irreversible colonic dilation and hypomotility. ${ }^{1}$ In cats, megacolon is most commonly idiopathic but may also be secondary to pelvic canal obstruction or neurologic trauma, and congenital megacolon has also been reported. ${ }^{2-4}$ Feline idiopathic megacolon is largely a diagnosis of exclusion and appears to be most common in overweight, middle-aged, male cats.,

Management of feline idiopathic megacolon is initially conservative, with the use of enemas, laxatives, and prokinetic agents to minimize colonic content retention and provision of stool softeners and dietary modifications to allow for easier evacuation. ${ }^{6,7}$ Unfortunately, medical management is often unrewarding in the long term, and surgical intervention with subtotal colectomy has been recommended as the definitive treatment of choice. ${ }^{6,8,9}$ However, controversy exists around subtotal colectomy in regards to outcome, complication rates, and whether removal of the ileocecocolic junction (ICJ) is warranted. Removal of the ICJ during subtotal colectomy was originally advocated to reduce tension on the anastomosis site and to reduce rates of recurrence. ${ }^{10}$ Preservation of the ICJ has since been endorsed to reduce complications such as intractable diarrhea secondary to small intestinal bacterial overgrowth., ${ }^{, 11}$ 
There is a paucity of literature on subtotal colectomy for the treatment of cats with idiopathic megacolon, particularly concerning outcomes, complication rates, recurrence rates, and the effect of removal of the ICJ. Previous studies ${ }^{5,12}$ have investigated long-term prognosis and negative prognostic factors for patients undergoing subtotal colectomy for treatment of idiopathic megacolon., ${ }^{5}$ However, there is conflicting information regarding the postoperative quality of life following surgery as perceived by the animal owners. ${ }^{5,12-14}$ Although 1 study $^{12}$ shows that most owners will tolerate their cat having soft feces and other complications, findings of other studies ${ }^{13,14}$ indicate that perineal soiling is a common sequela of liquid feces and is a major complaint of owners. Recurrence of constipation has been reported ${ }^{5}$ as high as $45 \%(10 / 22)$, with $18 \%(4 / 18)$ of cats euthanized because of recurrence.

The objectives of the study reported here were to evaluate outcomes in a large group of cats undergoing subtotal colectomy for the treatment of idiopathic megacolon and to determine whether removal versus nonremoval of the ICJ was associated with differences in outcome. Our null hypothesis was that outcome would not differ for cats that had versus had not had their ICJ removed.

\section{Materials and Methods}

In this retrospective cohort study, medical records databases at 18 referral and academic teaching hospitals were searched for records of cats that underwent subtotal colectomy for the treatment of idiopathic megacolon between January 1, 2000, and December 31, 2018. Identified cats with available medical records and owner contact information were eligible for the study. Cats were excluded if idiopathic megacolon was not diagnosed or they did not have subtotal colectomy. Subtotal colectomy was defined as resection of the colon with or without removal of the ICJ. ${ }^{4}$ Case recruitment and dissemination of a premade spreadsheet (Excel version 16.16.23; Microsoft Corp) for data input were conducted by email between May 23 to October 23, 2019.

\section{Data collection}

Preoperative data-We collected information on patient breed, sex, neuter status, age at the time of surgery (months), body weight (kg), body condition score (BCS) on a scale from 1 (extremely underweight) to 9 (extremely overweight), duration of constipation prior to presentation, and whether an inciting event was associated with the cat's constipation. Preoperative medical management data included information on the use of dietary management, prokinetic agents, stool softeners, and bulking agents and whether manual deobstipation, enemas, or both were performed. Additional data were collected on whether patients had concurrent comorbidities at the time of surgery and whether abnormalities were noted on preoperative blood work or urinalysis.
Surgical data-The surgical data collected included the date of surgery, surgical technique used for the subtotal colectomy anastomosis (eg, the use of continuous suture pattern, interrupted suture pattern, intestinal stapling equipment, or biodegradable anastomotic rings), whether the ICJ was removed, and whether the cranial rectal artery was ligated. Cats that had both continuous and interrupted suture patterns used for the subtotal colectomy were classified as having had a continuous pattern performed.

Postoperative data-Postoperative data collected included postoperative antimicrobial administration, feces consistency while hospitalized, total duration of hospitalization, whether a feeding tube had been placed, and incidence of postoperative tenesmus. Feces consistency was defined as formed (clinically normal), soft (semiformed), or liquid. If the cat did not defecate while hospitalized, this was also recorded. Postoperative complications were recorded and defined as minor when they did not require surgical intervention (eg, superficial wound infection or inappetence) or major when they required revision surgery or resulted in the death of the patient. Other outcome measures collected included recurrence of constipation (defined as any episode after surgical intervention in which the patient was unable to defecate without medical or surgical intervention), duration from surgery to recurrence of constipation, whether medical management (dietary, medications, enemas, or manual deobstipation, alone or in combination) was needed to for postoperative constipation, and whether revision surgery was required. When revision surgery was required, surgical data and postoperative data were collected as described earlier. Postoperative antimicrobials were defined as any antimicrobials given by any route after completion of the surgery, including antimicrobials given in the hospital or prescribed for continued treatment at home.

Follow-up data-All owners were contacted for follow-up information between October 3 and November 20, 2019. Follow-up data were also obtained from owner interviews, referring veterinarian interviews, and medical records. Follow-up data included the date of last follow-up communication, long-term postoperative fecal consistency (ie, liquid, soft, or formed), whether the patient was alive or the date and cause of death, and whether the death was related to megacolon. Owners were interviewed to ascertain whether they would elect to have the surgery performed again, and how they would describe their cat's quality of life after surgery. Quality of life was defined as either poor (eg, persistent intolerable clinical signs or euthanasia because of the disease process), fair (eg, difficulty controlling complications with medication, but tolerable for the owner and patient), good (eg, no clinical signs with additional medical or dietary management), or excellent (eg, no clinical signs, without the need for medical or dietary management).

Inclusion in the survival analysis required either direct contact by telephone or email communication with the 
owner, or a record that the patient had died with the cause of death noted to be either related or unrelated to the diagnosis of megacolon. Cats for which this information was not available were excluded from survival analysis.

\section{Statistical analysis}

Continuous data were assessed for normality with the Shapiro-Wilk test. Normally distributed data were reported as the mean $\pm \mathrm{SD}$, and nonnormally distributed data were reported as the median and interquartile (25th to 75 th percentile) range (IQR). A proportions test was used to evaluate sex predisposition for undergoing subtotal colectomy. Simple univariable logistic regression was used to evaluate for associations between study variables and binomial outcomes, including death within 10 days of surgery, presence of long-term liquid feces, recurrence of constipation, or good or excellent quality of life. Values of $P<0.05$ were considered significant.

Univariable Cox regression estimates were performed to evaluate survival time from completion subtotal colectomy for all factors, and Kaplan-Meier survival curves were generated for factors with values of $P<0.05$. Hazard ratios (HRs) with $95 \%$ CIs and $P$ values were reported. Patients that were alive at the end of study period or known to have died from a cause unrelated to feline idiopathic megacolon were right censored. All statistical calculations were performed with commercial software (version 15.1; StataCorp).

\section{Results}

\section{Animals}

There were 182 cats identified as having undergone subtotal colectomies for megacolon at 18 institutions during the study period. Of these patients, 8 had concurrent neoplasia, 1 had a distal colonic stricture of unknown cause, and 7 had substantial neurologic disorders or caudal spinal abnormalities. These 16 cats were excluded, leaving the records of the remaining 166 cats for evaluation.

The included cats were most commonly reported as domestic shorthair $(\mathrm{n}=102)$, domestic longhair (24), Siamese (10), or domestic medium hair (9). The remaining 21 cats were classified as Manx $(\mathrm{n}=5)$, Maine Coon (5), mixed-breed cats (4), tabby (2), Bengal (1), Nebelung (1), Cornish Rex (1), Persian (1), or Ragdoll (1). On the day of surgery, the median age and body weight were 8.6 years (IQR, 5.8 to 11.3 years; $\mathrm{n}=166$ cats) and $5.3 \mathrm{~kg}$ (IQR, 4.2 to $6.5 \mathrm{~kg} ; 165]$ ), respectively, and the mean \pm SD body BCS was $5.9 \pm 0.2(108)$. Cats were predominantly castrated males $(119 / 166[71.7 \%])$ or spayed females ( 47 [28.3\%]), with 1 (0.6\%) sexually intact male and $2(1.2 \%)$ sexually intact females also included. Male cats were 2.5 times as likely to have undergone a subtotal colectomy for idiopathic megacolon (OR, 2.5; 95\% CI, 1.86 to $3.76 ; P<0.001)$, compared with female cats.

\section{Preoperative data}

Severe constipation or obstipation was the primary clinical sign leading to presentation in all cats, and the median preoperative duration of clinical signs was 24 months (IQR, 12 to 42 months; $\mathrm{n}=162$ cats). Information on preoperative medical treatment was available for 166 cats, and treatments included manual deobstipation, enemas, or both (150 [90.4\%]); stool softeners (146 [88.0\%]); prokinetic agents (137 [82.5\%]); dietary modifications (83 [50.0\%]); or bulking agents ( $40[24.1 \%]$ ), alone or in combination. Additionally, other treatments (acupuncture, IV or SC administration of fluids, or administration of probiotics, mineral oil, antimicrobials, or holistic supplements, alone or in combination) were provided to $32(19.3 \%)$ cats. The presence or absence of comorbidities was noted in the medical records of 162 cats, with $40(24.7 \%)$ cats having had 1 or more comorbidities, including cardiac disease $(n=25[15.4 \%])$, renal disease (17 [10.5\%]), endocrine disease ( 7 [4.3\%]), or portosystemic shunt (4 $[2.5 \%])$.

\section{Surgical data}

Anastomotic technique for subtotal colectomy was reported for 155 cats and included the use of a simple interrupted suture pattern $(n=120$ [77.4\%]), simple continuous suture pattern (28 [18.1\%]), biodegradable anastomotic ring (5 [3.2\%]), or a circular end-to-end stapling device (2 [1.3\%]). The ICJ was removed from 41 of 160 (25.6\%) cats, the cranial rectal artery was ligated in 17 of 64 (26.6\%) of cats, and a feeding tube was placed in 21 of 165 (12.7\%) cats.

\section{Postoperative data}

Antimicrobials were administered postoperatively to 125 of 165 (75.8\%) cats. The median duration of hospitalization was 3 days (IQR, 2 to 4 days; $n=162$ cats).

\section{Outcomes of interest}

Postoperative complications-Major complications occurred for 15 of 151 (9.9\%) cats, and minor complications occurred for 75 (49.7\%) cats. Major complications included cardiopulmonary arrest within 10 days of surgery $(n=4)$, colectomy dehiscence (3), abdominal incision dehiscence (2), postoperative renal failure (1), septic peritonitis of unconfirmed etiology (1), postoperative necrosis of the rectal wall (1), retained surgical sponge (1), sudden development of a mass dorsal to the colectomy site causing compression over the anastomosis site (1), and persistent hematochezia requiring colonoscopy (1). Two cats underwent surgical correction for colectomy dehiscence: 1 cat on day 4 and the other cat on day 9 after surgery. The subtotal colectomy dehiscence rate was $2.1 \%(3 / 142)$ for cats that underwent follow-up for $\geq 10$ days after surgery. Various minor complications were encountered, with anorexia or inappetence accounting for $72 \%(54 / 75)$.

Death within 10 days after surgery-For cats with $\geq$ 10 days of postsurgical follow-up information, the 10day perioperative death rate was 5.6\% (8/142). Cats had greater odds of death within the first 10 days after surgery if they had a BCS < 4/9 (OR, 19.25; 95\% CI, 3.04 
to $122.1 ; P=0.002)$, preoperative cardiac disease (OR, $10.5 ; 95 \% \mathrm{CI}, 2.30$ to $47.6 ; P=0.002)$, postoperative tenesmus (OR, 8.88; 95\% CI, 1.49 to $52.7 ; P=0.016$ ), or major complication (OR, 115; 95\% CI, 12.4 to 1,$069 ; P$ $<0.001)$. There were no meaningful associations identified between the outcome of death within 10 days after surgery and the other variables evaluated, including age, duration of preoperative clinical signs, preoperative kidney disease, preoperative endocrinopathies, removal of the ICJ, placement of a feeding tube, use of postoperative antimicrobials, presence of liquid feces immediately postoperatively, and minor complications.

\section{Fecal consistency}

The medical records of 144 cats described the patient's fecal consistency immediately after surgery as either liquid (60 [41.7\%]), soft (43 [29.9\%]), or formed (21 [14.6\%]) or indicated that the patient had not defecated between surgery and hospital discharge (20 [13.9\%]). Postoperative tenesmus was observed in 25 of 111 (22.5\%) cats.

The final fecal consistency reported during a follow-up interview with the owner or the last veterinary evaluation was soft or formed for 100 of 121 (82.6\%) cats or liquid for the remaining 21 (17.4\%) cats. Cats from which the ICJ had been removed had significantly $(P=0.014)$ greater odds of long-term liquid feces (OR, 3.45; 95\% CI, 1.29 to 9.28) than did cats from which the ICJ had not been removed. However, the median follow-up duration was significantly $(P<$ $0.001)$ shorter for cats with liquid feces (64 days; IQR, 20 to 503 days) versus cats with either formed or soft feces (740 days; IQR, 145 to 1,908 days).

\section{Recurrence of constipation}

Recurrence of constipation was reported for 24 of $74(32 \%)$ cats that met the follow-up criteria, and

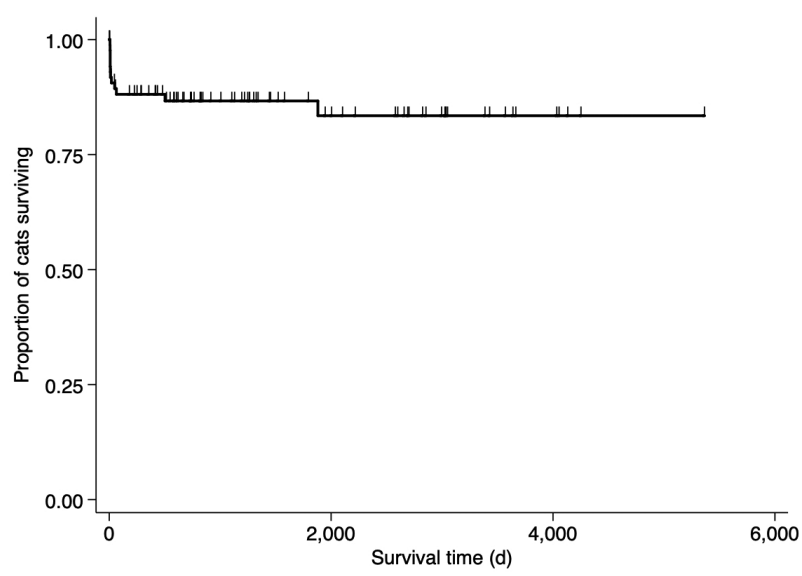

Figure I-Kaplan-Meier curve of survival time for 87 cats after subtotal colectomy between January I, 2000, and December 3I, 2018, for treatment of idiopathic megacolon; cats met the follow-up criteria for inclusion in survival time analysis. Tick marks indicate censored cats; steps represent the death of $\geq$ I cat attributed to megacolon-related causes ( $n=$ 12). The median survival time was not reached. the median duration from surgery to recurrence of constipation was 344 days (IQR, 47 to 1,159 days). Affected cats were managed with medications (20/21 [95\%]), dietary modification (11/21 [52\%]), enemas (10/20 [50\%]), manual deobstipation (7/20 [35\%]), or revision surgery $(3 / 24$ [13\%]). The 3 patients that underwent revision surgery did so at 33, 410, and 2,097 days after their first subtotal colectomy. No meaningful association was detected between recurrence of constipation and nonremoval of the ICJ during the initial subtotal colectomies (OR, 1.42; 95\% CI, 0.40 to $5.02 ; P=0.59$ ) or any of the other variable.

\section{Survival time analysis}

Eighty-seven of the 166 (52.4\%) cats met the stringent conditions for inclusion in the survival time

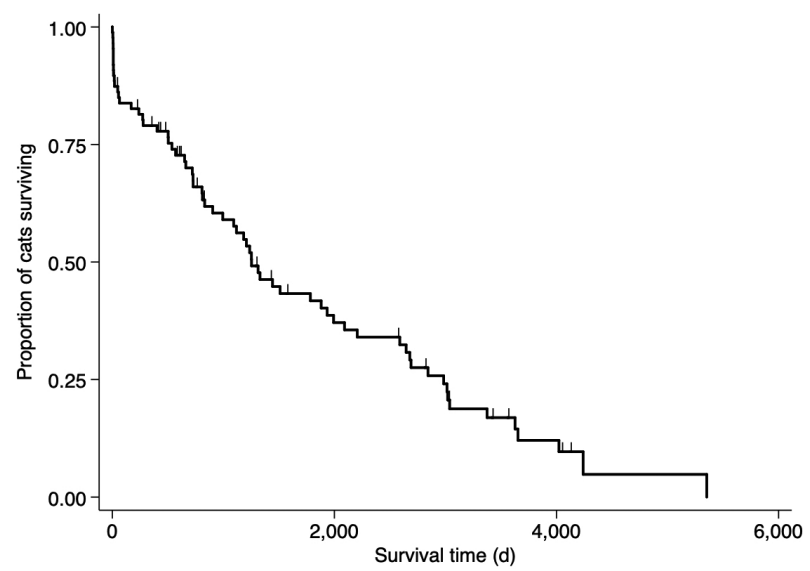

Figure 2-Kaplan-Meier curve of survival time for 65 cats described in Figure I that died of any cause after subtotal colectomy. Tick marks indicate censored cats; steps represent the death of $\geq 1$ cat. The overall median survival time was I,254 days ( $95 \% \mathrm{Cl}, 904$ to I,935 days), and the maximum survival time was 5,353 days.

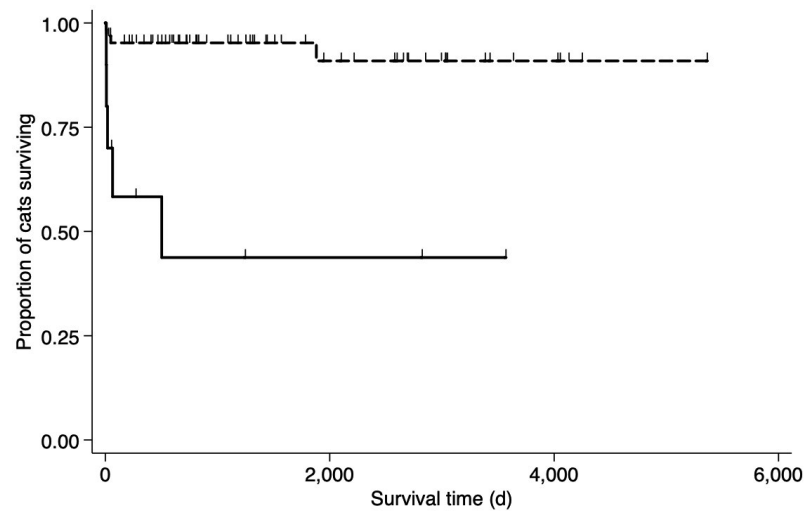

Figure 3-Kaplan-Meier curves of survival time for 74 cats described in Figure I with $(\mathrm{n}=10$; solid line) versus without (64; dashed line) long-term liquid feces postoperatively. Tick marks indicate censored cats; steps represent the death of $\geq$ I cat. Cats with long-term liquid feces postoperatively had a greater hazard of shorter survival time (hazard ratio, 10.4; $95 \% \mathrm{Cl}, 2.77$ to $39.0 ; P=0.00 \mathrm{I}$ ). 
analysis. On follow-up interview, 12 of these 87 (14\%) cats were confirmed to have died or have been euthanized because of complications associated with idiopathic megacolon, and 22 (25\%) cats were still alive. When cats that died or were euthanized for reasons only associated with idiopathic megacolon were considered, a median survival time (MST) was not reached (Figure I). The overall MST was 1,254 days (95\% CI, 904 to 1,935 days) for all 87 cats, with a maximum lifespan of 5,353 days (Figure 2). Variables associated with greater hazard of shorter survival time (Figure 3; Table I) were BCS < 4/9 (HR, 5.97; 95\%
CI, 1.32 to $18.7 ; P=0.017)$, preexisting heart disease (HR, 3.21; 95\% CI, 1.01 to $0.2 ; P=0.048$ ), major complication (HR, 27.8; 95\% CI, 7.88 to $98.0 ; P<0.001$ ), or long-term liquid feces (HR, 10.4; 95\% CI, 2.77 to $39.0 ; P=0.001)$.

\section{Owner assessment}

Owner data were not available for all variables. Sixty-five of 72 (90\%) owners indicated that they were happy with the decision to pursue surgery. Seventy-four owners reported that they perceived their cat's quality of life after surgery was excellent

Table I-Univariable Cox regression analysis to identify variables that negatively affected survival time in 87 cats that underwent subtotal colectomy for treatment of idiopathic megacolon between January I, 2000, and December 3I, 2018, and that met the follow-up criteria for inclusion in survival time analysis.

\begin{tabular}{|c|c|c|c|}
\hline Variable & No. of cats & $\begin{array}{c}\text { Hazard ratio } \\
(95 \% \mathrm{CI})\end{array}$ & $P$ value \\
\hline Sex & 87 & & \\
\hline Male & 61 & I.32 (0.36-4.86) & 0.680 \\
\hline Female & 26 & Referent & \\
\hline Increasing age (mo) & 87 & $1.00(0.99-1.02)$ & 0.501 \\
\hline BCS & 54 & & \\
\hline$<4$ & 10 & $5.97(1.32-18.7)$ & 0.017 \\
\hline$\geq 4$ & 44 & Referent & \\
\hline Increasing duration of preoperative clinical signs & 85 & $0.97(0.94-1.01)$ & 0.138 \\
\hline Preoperative heart disease & 86 & & \\
\hline Yes & 20 & $3.21(1.01-10.2)$ & 0.048 \\
\hline No & 66 & Referent & \\
\hline Preoperative kidney disease & 86 & & \\
\hline Yes & 10 & $0.78(0.10-6.08)$ & 0.816 \\
\hline No & 76 & Referent & \\
\hline Preoperative endocrine disease & 86 & & \\
\hline Yes & 4 & $2.33(0.30-18.1)$ & 0.418 \\
\hline No & 82 & Referent & \\
\hline Suture pattern for anastomosis & 74 & & \\
\hline Simple interrupted & 59 & $|.4|(0.3 \mathrm{I}-6.45)$ & 0.657 \\
\hline Simple continuous & 15 & Referent & \\
\hline ICJ removal & 83 & & \\
\hline Yes & 17 & $\mathrm{I} .50(0.40-5.56)$ & 0.548 \\
\hline No & 66 & Referent & \\
\hline Postoperative tenesmus & 60 & & \\
\hline Yes & 16 & $3.78(0.9|-| 5.7)$ & 0.068 \\
\hline No & 44 & Referent & \\
\hline Postoperative feeding tube placement & 86 & & \\
\hline Yes & 8 & $2.40(0.52-11.0)$ & 0.260 \\
\hline No & 78 & Referent & \\
\hline Postoperative antimicrobial administration & 86 & & \\
\hline Yes & 63 & $0.54(0.17-1.72)$ & 0.299 \\
\hline No & 23 & Referent & \\
\hline Liquid feces in the immediate postoperative period & 69 & & \\
\hline Yes & 32 & $\mathrm{I} .52(0.4 \mathrm{I}-5.67)$ & 0.531 \\
\hline No & 37 & Referent & \\
\hline Major complications & 83 & & \\
\hline Yes & II & $27.8(7.88-98.0)$ & $<0.001$ \\
\hline No & 72 & Referent & \\
\hline Minor complications & 83 & & \\
\hline Yes & 45 & $1.25(0.40-3.94)$ & 0.705 \\
\hline No & 38 & Referent & \\
\hline Recurrence of constipation & 74 & & \\
\hline Yes & 24 & $0.82(0.2 I-3.17)$ & 0.769 \\
\hline No & 50 & Referent & \\
\hline Revision surgery & 74 & & \\
\hline Yes & 3 & $3.04(0.38-24.0)$ & 0.293 \\
\hline No & 7I & Referent & \\
\hline Identification of long-term liquid feces & 74 & & \\
\hline Yes & 10 & $10.4(2.77-39.0)$ & 0.001 \\
\hline No & 64 & Referent & \\
\hline
\end{tabular}

BCS = Body condition score. $I C J$ = lleocecocolic junction 
$(n=28[38 \%]), \operatorname{good}(29[39 \%])$, fair $(7$ [9\%]), or poor (10 [14\%]). Cats with long-term liquid feces had 11.4 times the odds (OR, 11.4; 95\% CI, 2.70 to $47.8 ; P=$ 0.001 ) of having had either a fair or poor quality of life score reported by their owners than did cats with soft or firm feces. Cats from which ICJs had been removed had 3.6 times the odds (OR, 3.6; 95\% CI, 1.02 to $12.7, P=0.046)$ of their owners reporting either a fair or poor quality of life score, compared with cats in which the ICJs had not been removed.

\section{Discussion}

Results indicated that, despite recurrence of constipation in 24 of 74 (32\%) cats in this large multicenter retrospective cohort study, cats that undergo subtotal colectomy for idiopathic megacolon can experience long survival times with a high degree of owner satisfaction. Overall, $77 \%$ (57/74) of owners in the present study determined that the quality of life of their cat following surgery was good (required daily medication or dietary management) or excellent (had no clinical signs and required no medication or dietary management). Retention of the ICJ was not associated with recurrence of constipation; however, a significant association was found between removal of the ICJ and the development of long-term liquid feces. Furthermore, cats that underwent ICJ removal were more likely to have a fair to poor outcome as determined by owner assessment. Because the presence of long-term liquid feces was also associated with shorter survival time and lower owner-assigned quality of life scores, we rejected our null hypothesis that removal of the ICJ does not lead to differences in outcome. Results of the present study provided support for not removing the ICJ during subtotal colectomy for the treatment of idiopathic megacolon in cats.

Diarrhea is a common and somewhat expected sequela for cats undergoing subtotal colectomy.3,10,12,13 The colon has a substantial role in the removal of water from the feces and in maintaining electrolyte balance; therefore, removal of the colon reduces these capacities. ${ }^{1,15}$ An experimental study ${ }^{14}$ of cats undergoing subtotal colectomy shows that the ileum adapts over 2 months to increase water resorption by increasing villus height and density. As a result, cats tend to have 2 to 8 weeks of diarrhea following surgery that should theoretically improve over time. However, a proportion of these cats will continue to have diarrhea, which may be severe, for the rest of their lives. $^{2,5}$ Although soft feces are often well tolerated by owners, continued passage of liquid feces can be distressing because it leads to perineal soiling and irritation, necessitating frequent bathing. ${ }^{13,14}$ Some owners seem to cope with this postoperative complication, whereas others seem not to and may elect euthanasia for the cat.5,12 This was supported by the results of our study in which cats with, versus without, long-term liquid feces had 11.4 times the odds of only fair or poor quality-of-life score assessment by their owners. Although $41.7 \%(60 / 144)$ of the cats in the present study had liquid feces immediately after surgery, the final recorded fecal consistency on follow-up showed improvement, with liquid feces reported in only $17.4 \%(21 / 121)$ of cats. Despite this, a significant hazard of shorter survival time was associated with the presence of liquid feces at follow-up, which could have indicated that this complication was poorly tolerated by owners.

A significant association was found between removal of the ICJ and the presence of liquid feces reported on follow-up in the present study. In a previous study ${ }^{5}$ of 22 cats undergoing subtotal colectomy for idiopathic megacolon, removal, versus retention, of the ICJ was significantly associated with long-term looser feces. Given the negative association between the presence of liquid feces at follow-up and outcome, understanding the role of the ICJ in fecal consistency is essential for operative decision-making; however, the importance and ultimate function of the ICJ are debated in the literature. ${ }^{1,3,5,11,15}$ For instance, the ICJ is thought to act as a barrier to the invasion of the small intestine by colonic bacteria, and removal of the ICJ can result in small intestinal bacterial overgrowth that consequently leads to deconjugation of bile acids, fat malabsorption, and steatorrhea. ${ }^{15}$ Investigators ${ }^{3,5}$ propose maintaining the ICJ during subtotal colectomy to reduce the occurrence of severe diarrhea and thus to improve outcome. ${ }^{3,5}$ Conversely, removal of the ICJ has been advocated because doing so allows resection of the proximal portion of the colon, which is thought by some to reduce recurrence. ${ }^{1,11}$ In addition, removal of the ICJ reduces tension on the anastomosis site owing to the increased mobility of the distal segment of the small intestine and potentially less technically demanding surgery. ${ }^{1,5,11}$ We identified no association between the recurrence of constipation and either preservation or removal of the ICJ in the present study, consistent with other reports.,14 The dehiscence rate of $2.1 \%(3 / 142)$ was too low to perform a meaningful evaluation between the effect of anastomotic tension and dehiscence. Therefore, given that removal of the ICJ was associated with an increased likelihood of long-term liquid feces with decreased quality-of-life scores without reducing the incidence of constipation recurrence in the present study, we recommend preservation of the ICJ during subtotal colectomy for idiopathic megacolon.

Recurrence of constipation in the present study was reported for $32 \%(24 / 74)$ cats at a median of 344 days, which was comparable with previously reported rates of $45 \%(10 / 22))^{5}$ Despite this, the reoperation rate in the present study was low. For most cats with recurrence of constipation in the present study, the issue was controlled with medical management (eg, dietary changes, prokinetics, stool softeners, enemas, or manual deobstipation) rather than surgery, consistent with previous reports.,10,11 This may be attributable to a general reluctance of owners to pursue a second surgery or the shortened colon being more amenable to medical management following the procedure. The cause of recurrence in cats un- 
dergoing subtotal colectomy for idiopathic megacolon is generally unknown, and we did not identify a significant association with recurrence and any of the variables evaluated in the present study. Although the pathogenesis of this disease remains undetermined, a generalized disorder of colonic smooth muscle is postulated. ${ }^{6}$ Therefore, although unproven, it is plausible that recurrence of constipation occurs in the remaining portion of the diseased colon. Preservation of the ICJ necessitates, leaving 2 to $3 \mathrm{~cm}$ of proximal ascending colon in contrast to removal of the ICJ. Removal of the distalmost portion of the colon requires ligation of the cranial rectal artery, ${ }^{16}$ which was performed in only $25.6 \%$ (41/160) of cats in the present study. Even when removing both the ICJ and distal colon, a cuff of the large intestine must remain to allow for the anastomosis, which limits the ability to achieve a true total colectomy. ${ }^{16}$ This irrevocable necessity to leave a portion of the colon may underly the reason that the present study and others have been unable to identify an association between removal of the ICJ, or distal colon with cranial rectal artery ligation, and a decreased rate of constipation recurrence. ${ }^{5,9,14}$ A prospective study quantifying the length of the remaining colon would be required to better answer the question of how much colon should be removed to prevent disease recurrence, if this is indeed the underlying cause of this complication.

An MST could not be identified for the cats in the present study, a finding supportive for cats undergoing surgical treatment for idiopathic megacolon and having the potential for long survival times. For cats that met the criteria for inclusion in survival time analysis in the present study, 14\% (12/87) died of megacolon-related causes. Two-thirds $(8 / 12)$ of these cats died $\leq 10$ days after the initial surgery (perioperative death rate of $5.6 \%[8 / 142]$ ) as a result of a major complication. Death $\leq 10$ days of surgery in the present study was significantly associated with a BCS $<4 / 9$, the presence of preoperative heart disease, and postoperative tenesmus. Variables with a greater hazard of shorter survival time included preexisting heart disease, development of major complications, BCS < $4 / 9$, and long-term liquid feces. Although associations with the development of major complications (those that required reoperation or resulted in death) and survival time are obvious, other associations may be less so. Preexisting heart disease can make postoperative care in cats challenging, particularly regarding fluid balance. ${ }^{17}$ Postoperative tenesmus and long-term liquid feces are complications that are often not tolerated by owners and associated with a poor quality of life..$^{5}$ A poor BCS is likely a reflection of an inability to maintain body weight properly prior to surgery because of the development of either a cachectic state or underlying disease. Cachexia can alter important molecules in the body, such as inflammatory mediators, catecholamines, and cortisol, which are important factors in wound healing, strength, immune function, the ability to metabolize fat as an alternate energy source, and survival. ${ }^{18,19}$ Further investigation is warranted to understand the pathophysiologic processes that could have contributed to the association between a low BCS and both perioperative death and long-term survival times for cats of the present report. On the basis of our results, it may be prudent to carefully manage and supplement caloric intake in underweight cats prior to and after subtotal colectomy and to consider intervening earlier in cats losing substantial body weight.

There were several different surgical techniques used to perform the anastomosis in the present study, including the use of sutures (simple continuous or simple interrupted pattern), intestinal staplers, and biofragmentable anastomotic rings. Suturing was by far the most common technique used, accounting for $95.5 \%(148 / 155)$ of the techniques used to perform the anastomosis of all surgeries performed in the present study. The intestinal dehiscence rate in cats with at least 10 days of follow-up was very low (2.8\% [4/142]). This finding was in contrast to a popularly held belief that large intestinal surgery is associated with higher dehiscence rates than small intestinal surgery. ${ }^{4}$ A recent study ${ }^{20}$ evaluating full-thickness large intestinal incisions in 90 dogs shows a dehiscence rate of $10 \%$ (9/90). That study ${ }^{20}$ also indicates associations between intestinal dehiscence and the administration of blood products, administration of more than 2 classes of antimicrobials, and presence of septic peritonitis. The low rate of intestinal dehiscence in the present study precluded meaningful associations between dehiscence and surgical and postoperative variables, including surgical technique. Thus, when subtotal colectomy is properly performed, the risk of intestinal dehiscence should not be considered a contraindication for the procedure in cats with idiopathic megacolon.

Although the present study provided information on a large number of cats with idiopathic megacolon, its retrospective, multi-institutional nature also led to several limitations, including incomplete medical record information, inconsistencies in surgical and medical management protocols and record documentation, and a lack of needed follow-up information for all cases. Additionally, the definition of fecal consistency was somewhat subjective, whereas ideally an established fecal scoring system would be used. As with other retrospective studies, bias was likely reported by owners who were either very satisfied or very dissatisfied. The long study period that allowed for the large sample size also led to a loss of data either through purging of records or inaccurate owner recall for patients that had died earlier. Further, the follow-up period for cats with versus without liquid feces was different in that some cats with liquid feces had follow-up durations of $<8$ weeks. Given that the consistency of liquid feces is expected to improve with time, bias created by inequitable follow-up could have affected results for a relationship between liquid diarrhea and removal of the ICJ. However, the disparate follow-up periods could have been an accurate representation, given that cats with liquid feces had shorter survival times and poorer owner-assigned quality of life scores. Moreover, the 
median follow-up duration was 64 days for cats with liquid feces, which was longer than the expected 2- to 8-week duration for the colon to adapt and for fecal consistency to improve. ${ }^{14}$

To our knowledge, the present study contained the largest cohort of cats undergoing subtotal colectomy for treatment of idiopathic megacolon, and despite limitations, our findings suggested that subtotal colectomy is a reasonable treatment option, given the long survival times and high degree of owner satisfaction. On the basis of our results, we recommend that veterinarians do not remove the ICJ when performing subtotal colectomy in cats with idiopathic megacolon and that veterinarians provide additional attention to affected cats with a low BCS or preexisting heart disease, given the negative associations between these conditions and perioperative and long-term survival time.

\section{Acknowledgments}

The authors declare that there were no conflicts of interest

The authors thank Carol Frederick, Dr. Juliany Gomes Quitzan, Dr. Laura Selmic, and Dr. Junxian Zheng for their assistance with this manuscript.

\section{References}

1. Bertoy RW. Megacolon in the cat. Vet Clin North Am Small Anim Pract. 2002;32(4):901-915.

2. Webb SM. Surgical management of acquired megacolon in the cat. J Small Anim Pract. 1985;26(7):399-405.

3. White RN. Surgical management of constipation. J Feline Med Surg. 2002;4(3):129-138.

4. Williams JM. Colon. In: Tobias KM, Johnson SA, eds. Veterinary Surgery: Small Animal. Saunders Elsevier; 2012:1557-1561.

5. Sweet DC, Hardie EM, Stone EA. Preservation versus excision of the ileocolic junction during colectomy for megacolon: a study of 22 cats. J Small Anim Pract. 1994;25(7):358-363.

6. Washabau RJ, Hasler AJ. Constipation, obstipation and mega- colon. In: August JR, ed. Consultations in Feline Internal Medicine. 3rd ed. WB Saunders Co; 1997:104-112.

7. Hall EJ. Diseases of the large intestine. In: Ettinger SJ, Feldman EC, Côté E, eds. Textbook of Veterinary Internal Medicine. 8th ed. Elsevier Inc; 2017:1563-1592.

8. Trevail T, Gunn-Morre D, Carrera I, Courcier E, Sullivan M. Radiographic diameter of the colon in normal and constipated cats and in cats with megacolon. Vet Radiol Ultrasound. 2011;52(5):516-520.

9. Rosin E. Megacolon in cats. The role of colectomy. Vet Clin North Am Small Anim Pract. 1993;23(3):587-594.

10. Rosin E, Walshaw R, Mehlhaff R, Matthiesen D, Orsher R, Jusba J. Subtotal colectomy for treatment of chronic constipation associated with idiopathic megacolon in cats: 38 cases (1979-1985). J Am Vet Med Assoc. 1988;193(7):850-853.

11. Bright RM. Idiopathic megacolon in the cat: subtotal colectomy with preservation of the ileocolic valve. Vet Med Rep. 1991;3:183-187.

12. de Hann JJ, Ellison GE, Bellah JR. Surgical correction of idiopathic megacolon in cats. Feline Pract. 1992;20(2):6-11.

13. Ellison GW. Complications of gastrointestinal surgery in companion animals. Vet Clin North Am Small Anim Pract. 2011;41(5):915-934.

14. Bertoy RW, MacCoy DM, Wheaton LG, Gelberg HB. Total colectomy with ileorectal anastomosis in the cat. Vet Surg. 1989;18(3):204-210.

15. Gregory CR, Guilford WG, Berry CR, Olsen J, Pederson NC. Enteric function in cats after subtotal colectomy for treatment of megacolon. Vet Surg. 1990;19(3):216-220.

16. Bright RM, Burrows CF, Goring R, Fox S, Tilmant L. Subtotal colectomy for treatment of acquired megacolon in the dog and cat. J Am Vet Med Assoc. 1986;188(12):1412-1416.

17. Byers CG. Crystalloid and colloid fluid therapy. In: Ettinger SJ, Feldman EC, Côté E, eds. Textbook of Veterinary Internal Medicine. 8th ed. Elsevier Inc; 2017:536-543.

18. Freeman L. Cachexia and sarcopenia: emerging syndromes of importance in dogs and cats. J Vet Intern Med. 2012;26(1):3-17.

19. Anker SD, Ponikowski P, Varney S, et al. Wasting as independent risk factor for mortality in chronic heart failure. Lancet. 1997;349(9058):1050-1053.

20. Latimer CR, Lux CN, Grimes JA, et al. Evaluation of shortterm outcomes and potential risk factors for death and intestinal dehiscence following full-thickness large intestinal incisions in dogs. J Am Vet Med Assoc. 2019;255(8):915-925. 